\title{
FAULT EVALUATION OF UNMANNED
}

\section{AERIAL VEHICLES POWER SYSTEM WITH AN IMPROVED FUZZY GROUP DECISION-MAKING}

\author{
Yuehao Yan, ${ }^{*}$ Zhiying Lv, ${ }^{* * * * *}$ and Ruiyu Zhang****
}

\begin{abstract}
The applications of unmanned aerial vehicles (UAVs) in military and civilian service domains have exhibited unprecedented growth in the last decades. However, the sophisticated and expensive UAVs are susceptible to multiple faults, such as wear and tear, noise, or software-control failures. The mutual recognition of community opinion in fuzzy multiple attribute group decisionmaking (FMAGDM) is an efficient way to solve a complex system. However, whether it can be utilized to evaluate the fault of the power system of UAVs has not been examined yet. This paper studies the evaluation towards the fault of the power system of UAVs under interval-valued intuitionistic fuzzy (IVIF) numbers with an improved FMAGDM. A new algorithm is proposed to achieve a desirable consensus in group decision-making. In contrast to the commonly used method, the proposed method comprehensively evaluates the fault of the power system of UAVs and basically gives an optimal consensus decision-making without modifying many elements.
\end{abstract}

\section{Key Words}

Unmanned aerial vehicle (UAV), fault evaluation, fuzzy multiple attribute group decision-making (FMAGDM), interval-valued intuitionistic fuzzy (IVIF) number, consensus

\section{Introduction}

An unmanned aerial vehicle (UAV), sometimes also called an unmanned aerial system, specifically refers to an aircraft, or more generally a flying machine, being flown

* College of Mechanical Engineering, Chengdu Technological University, Chengdu, People's Republic of China; e-mail: yanyuehao@126.com

** College of Management, Chengdu University of Information Technology, Chengdu, People's Republic of China; e-mail: 229059014@qq.com

*** College of Mathematics, University of Electronic Science and Technology of China, Chengdu, People's Republic of China; e-mail:229059014@qq.com

**** Beijing Institute of Information Technology, Beijing, People's Republic of China; e-mail: z5104@163.com Corresponding author: Zhiying Lv

Recommended by Prof. Anmin Zhu

(DOI: 10.2316/Journal.206.2018.3.206-5359) without a human pilot on board actively directing and piloting. In contrast with traditional aircraft, UAVs are generally either automatically controlled by the sophisticated computer systems on board or remotely controlled by human pilots on the ground [1]-[2], or a combination of both in real time. UAVs have exhibited unprecedented growth in military and civilian application [3]-[7], because of the low-cost operation, rapid placement, safe access to dangerous areas, flexible and scalable deployment to achieve many tasks [8]. However, UAVs are susceptible to numerous physical and virtual faults such as wear and tear, noise, software-control failures, and so on. If not detected in time, some faults can quickly deteriorate into a catastrophe, causing great damage to the UAV itself or its surroundings. Just right the expensive cost of UAVs and the potential damage, the potential applications of UAV in some domains are greatly hampered. Furthermore, as the increasing complexity of real-time decision problem, decision makers are frequently challenged by how to make an optimal decision [9] in an uncertain context due to their unconscious preferences. Therefore, how to make an order of the faults through optimal decision-making methods is of urgent importance.

In real decision situations, the information may not be quantified, it may be presented by the terms of their degrees of acceptance and degree of rejection. Thus, the imprecise information can be represented by intuitionistic fuzzy numbers (IFS). Fuzzy decision-making [10] aimed at making decisions under complex and uncertain environments where the information can be assessed with fuzzy sets and systems. Fuzzy multiple attribute group decision-making (FMAGDM) is defined as a decision situation in which a solution alternative to a given question has to be chosen based on the information given by different people or experts. In general, FMAGDM models under interval-valued intuitionistic fuzzy numbers (IVIFS) should be divided into four processes, namely, aggregation process, similarity measures process, consensus process and selection process. As one of the main measures for modern decision science, FMAGDM will improve the effectiveness and transparency of emergency management through integrating the 
wisdom of multiple decision makers into group wisdom. In recent years, some multiple attribute decision-making [11]-[12] methods and some multiple attribute group decision-making (MAGDM) methods [13]-[15] based on IVIFS have been proposed.

Moreover, some unique elements such as the diversity of expert source and the distinct difference in professional background and knowledge structure usually cause some disadvantages of emergency decisions when utilizing FMAGDM rather than fuzzy decision-making. In the consensus process, numerous methodologies including metagame analysis [16], drama theory [17], and the graph model for conflict resolution [18] have been developed to assist in understanding, modelling and analysing the conflict. As a result, a method to represent and analyse conflict situations with at least two decision makers with multiple options and objectives can be provided. However, some necessary improvements are still needed to handle the conflict of group decision. During the last years, some momentous developments have gained regarding solving the conflicts of group decisions. For example, Xu et al. [19] realized that it is important to deal with large and complicated conflict problem with introducing a matrix representation based on the traditional status quo analysis. Zhang et al. [20] introduced a conflict resolution framework to solve the conflict of land-use planning based on a geographic information system. Besides, some consensus models including multiplicative preference relations [21], fuzzy preference relations [22]-[23] and linguistic preference relations [24] have been developed to solve the conflicts across experts in GDM. For example, Zografos et al. [25] presented a methodological framework for developing a hazardous material emergency response decision support system to manage emergency response operations for large-scale industrial accidents. Parreiras et al. [26] developed a flexible conflict-eliminating model for FAGDM under linguistic assessment to maximize the soft consensus index using a procedure to search the opinion weight of experts. Whereas whether similar decision-making methods can be utilized to evaluate the faults of UAV has not been explored yet.

In this paper, a fault evaluation of UAV power system based on a new FMAGDM method under the intervalvalued intuition environment is presented. A new optimal consensus method with the minimum sum of weighted distance among individual opinions is proposed. The main body of the paper is organized as follows. Firstly, the main concepts of IVIFS and FMAGDM are illustrated. Then, the consensus process is demonstrated. Furthermore, a fault evaluation of UAV power system is constructed to demonstrate the feasibility and suitability of the proposed method. Finally, the advantages and future directions of this method are provided based on its practical performance.

\section{FMAGDM Problem with IVIFS}

The basic concepts of the IVIF are briefly introduced as follows.

Definition 1. Let $X=\left\{x_{1}, x_{2}, \ldots, x_{n}\right\}$ be a universe of discourse. Then an IVIF set $\tilde{p}$ on $X$ is given by

$$
\tilde{p}=\left\{<x, \tilde{u}_{\tilde{p}}(x), \tilde{v}_{\tilde{p}}(x)>, x \in X\right\}
$$

where $\tilde{u}_{\tilde{p}}(x)$ and $\tilde{v}_{\tilde{p}}(x)$ denote interval-valued membership and non-membership degree of $x$ to $\tilde{p}$ such that $\tilde{u}_{\tilde{p}}(x) \subseteq[0,1], \tilde{v}_{\tilde{p}}(x) \subseteq[0,1]$ and $0 \leq \sup \left(\tilde{u}_{\tilde{p}}(x)\right)+$ $\sup \left(\tilde{v}_{\tilde{p}}(x)\right) \leq 1$, for $\forall x \in X$. Denote the complement of $\tilde{p}$ is

$$
\tilde{p}^{\mathrm{c}}=\left\{<x, \tilde{v}_{\tilde{p}}(x), \tilde{u}_{\tilde{p}}(x)>, x \in X\right\}
$$

For convenience, let $\tilde{u}_{\tilde{p}}(x)=[a, b]$ and $\tilde{v}_{\tilde{p}}(x)=[c, d]$, then $\tilde{p}=([a, b],[c, d])$ is an IVIF number.

Definition 2. Let $\tilde{p}=([a, b],[c, d])$ be an IVIF number, then the score function of $\tilde{a}$ is

$$
S(\tilde{p})=\frac{a+b-c-d}{2}
$$

and the accuracy function of $\tilde{p}$ is

$$
H(\tilde{p})=\frac{a+b+c+d}{2}
$$

For $\tilde{p}_{1}$ and $\tilde{p}_{2}$, if $S\left(\tilde{p}_{1}\right)>S\left(\tilde{p}_{2}\right)$, then $\tilde{p}_{1}>\tilde{p}_{2}$. If $S\left(\tilde{a}_{1}\right)=$ $S\left(\tilde{a}_{2}\right), \tilde{a}_{1}>\tilde{a}_{2}$ and $\tilde{a}_{1}=\tilde{a}_{2}$ can be deduced, respectively, from $H\left(\tilde{a}_{1}\right)>H\left(\tilde{a}_{2}\right)$ and $H\left(\tilde{a}_{1}\right)=H\left(\tilde{a}_{2}\right)$.

By this definition, the rank of IVIF numbers can be obtained on the basis of their score functions or accuracy functions.

Definition 3. Let $\left\{\tilde{p}_{1}, \tilde{p}_{2}, \cdots, \tilde{p}_{n}\right\}$ be IVIF numbers, where $\tilde{p}_{k}=\left\{\tilde{u}_{k}, \tilde{v}_{k}\right\}=\left(\left[a_{k}, b_{k}\right],\left[c_{k}, d_{k}\right]\right) \in \tilde{S}$. Suppose $\lambda=\left(\lambda_{1}, \lambda_{2}, \cdots, \lambda_{t}\right)$ is the associated weight vector, where $\lambda_{k} \geq 0$ and $\sum_{k=1}^{t} \lambda_{k}=1$. The IVIF weighted average (IVIFWA) operator is a mapping IVIF WA: $\Omega^{t} \rightarrow \Omega$ according to

$$
\begin{aligned}
\tilde{p} & =\operatorname{IVIFWA}\left(\tilde{p}_{1}, \tilde{p}_{2}, \cdots, \tilde{p}_{t}\right) \\
& =\lambda_{1} \tilde{p}_{1}+\lambda_{2} \tilde{p}_{2}+\cdots+\lambda_{t} \tilde{p}_{t}=(\tilde{u}, \tilde{v})
\end{aligned}
$$

where $\tilde{u}=\left[\sum_{k=1}^{t} \lambda_{k} a_{k}, \sum_{k=1}^{t} \lambda_{k} b_{k}\right], \tilde{v}=\left[\sum_{k=1}^{t} \lambda_{k} c_{k}\right.$, $\left.\sum_{k=1}^{t} \lambda_{k} d_{k}\right]$.

Definition 4. Let $\tilde{p}_{1}=\left(\tilde{u}_{1}, \tilde{v}_{1}\right)=\left(\left[a_{1}, b_{1}\right],\left[c_{1}, d_{1}\right]\right)$ and $\tilde{p}_{2}=\left(\tilde{u}_{2}, \tilde{v}_{2}\right)=\left(\left[a_{2}, b_{2}\right],\left[c_{2}, d_{2}\right]\right)$, then the deviation between $\tilde{p}_{1}$ and $\tilde{p}_{2}$ is

$$
d\left(\tilde{p}_{1}, \tilde{p}_{2}\right)=\left|a_{1}-a_{2}\right|^{2}+\left|b_{1}-b_{2}\right|^{2}+\left|c_{1}-c_{2}\right|^{2}+\left|d_{1}-d_{2}\right|^{2}
$$

Clearly, $0 \leq d\left(\tilde{p}_{1}, \tilde{p}_{2}\right) \leq 1 ; d\left(\tilde{p}_{1}, \tilde{p}_{2}\right)=0$ if and only if $\tilde{p}_{1}=\tilde{p}_{2}$.

The FMAGDM problem based on IVIF number can be presented as follows. Assume that $A=$ $\left\{A_{1}, A_{2}, \ldots, A_{n}\right\}(n \geq 2)$ is an alternative set, which is assessed on $m$ attributes. The set of all attributes 
is denoted by $C=\left\{c_{1}, c_{2}, \ldots, c_{m}\right\}(m \geq 2)$, where attributes are independent of each other. Suppose that $\omega=\left(\omega_{1}, \omega_{2}, \ldots, \omega_{m}\right)^{T}$ is the weight vector of attributes given to an attribute set $C$, such that $\sum_{j=1}^{m} \omega_{j}=1, \omega_{j} \geq 0$, where $\omega_{j}$ denotes the weight of attribute $c_{j}$. The set of experts is denoted by $E=\left\{e_{1}, e_{2}, \ldots, e_{t}\right\}$ and assume $\lambda=\left(\lambda_{1}, \lambda_{2}, \ldots, \lambda_{t}\right)$ is the weight vector of the experts, where $\lambda_{k} \in(0,1), \sum_{k=1}^{t} \lambda_{k}=1$. Suppose $\tilde{P}^{k}=\left(\tilde{p}_{i j}^{k}\right)_{n \times m}$ is the IVIF decision matrix given by the expert $e_{k}$, whereas $\tilde{p}_{i j}^{k}=\left(\tilde{u}_{i j}^{k}, \tilde{v}_{i j}^{k}\right)$ represents the performance of the alternative $A_{i}$ with respect to the attribute $c_{j}$.

\section{Consensus Control Process}

In this section, the degree of conflict between individual opinions and group opinion is defined. Then, a group consensus reaching process is introduced. In general, consensus measures are available tools to assess the consensus degree between individual opinions and the group opinion from the experts. Next, some essential definitions are given.

Definition 5. Let $\tilde{P}^{1}=\left(\tilde{p}_{i j}^{1}\right)_{n \times m}$ and $\tilde{P}^{2}=\left(\tilde{p}_{i j}^{2}\right)_{n \times m}$ be two IVIF decision matrices. Then, the deviation degree between $\tilde{P}^{1}$ and $\tilde{P}^{2}$ is defined as follows:

$$
d\left(\tilde{P}^{1}, \tilde{P}^{2}\right)=\frac{1}{n m} \sum_{i=1}^{n} \sum_{j=1}^{m} d\left(\tilde{p}_{i j}^{1}, \tilde{p}_{i j}^{2}\right)
$$

Definition 6. Let $\tilde{P}^{k}=\left(\tilde{p}_{i j}^{k}\right)_{n \times m} \quad(k=1,2, \ldots, t)$ be $t$ IVIF matrices, where $\tilde{p}_{i j}^{k}=\left(\tilde{u}_{i j}^{k}, \tilde{v}_{i j}^{k}\right)$. Suppose $\tilde{p}=\lambda_{1} \tilde{P}^{1} \oplus \lambda_{2} \tilde{P}^{2} \oplus \cdots \oplus \lambda_{t} \tilde{P}^{t}$ is the group IVIF decision matrix using the IVIFWA operator, where $\tilde{P}=$ $\left(\tilde{p}_{i j}\right)_{n \times m}$, and $\tilde{p}_{i j}=\left(\sum_{k=1}^{t} \lambda_{k} \tilde{u}_{i j}^{k}, \sum_{k=1}^{t} \lambda_{k} \tilde{v}_{i j}^{k}\right)$.

Definition 7. Let $\tilde{P}^{k}=\left(\tilde{p}_{i j}^{k}\right)_{n \times m} \quad(k=1,2, \ldots, t)$ and be the kth decision matrix and the group decision matrix, respectively. Then, the group conflict degree for $\tilde{P}^{k}$ is defined by

$$
\theta\left(\tilde{P}^{k}\right)=d\left(\tilde{P}^{k}, P\right)=\frac{1}{n m} \sum_{i=1}^{n} \sum_{j=1}^{m} d\left(\tilde{p}_{i j}^{k}, \tilde{p}_{i j}\right)
$$

If $\theta\left(\tilde{P}^{(k)}\right)=0$, then the $k$ th expert has full consensus with the group opinions. Otherwise, the smaller the value of $\theta\left(\tilde{P}^{(k)}\right)$ is, the closer the expert is in agreement with the group. From the actual situation, the threshold $\delta$ is established by experts.

If $\forall k, \theta\left(\tilde{p}^{(k)}\right) \leq \delta$, the group reaches an desirable level of conflict, and thus decision makers have reached a consensus.

Let $\tilde{P}=\left\{\tilde{p}_{1}, \tilde{p}_{2}, \ldots, \tilde{p}_{t}\right\}$ be a set of IVIF variables, where $\tilde{p}_{k}=\left(\tilde{u}_{k}, \tilde{v}_{k}\right)$ and $\lambda=\left(\lambda_{1}, \lambda_{2}, \ldots, \lambda_{t}\right)^{T}$ be the associated weighting vector such that $\lambda_{k} \in[0,1]$ and $\sum_{k=1}^{t} \lambda_{k}=1$, the distance of each element $\tilde{p}_{k}$ in $\tilde{P}$ from the mean $\tilde{p}$ by $d\left(\tilde{p}_{k}, \tilde{p}\right)$ can be measured according to Definition 4. Then, we get the following definition.
Definition 8. The weighted distance of $\tilde{P}=\left\{\tilde{p}_{1}, \tilde{p}_{2}\right.$, $\left.\ldots, \tilde{p}_{t}\right\}$ is expressed as follows:

$$
S=\sum_{k=1}^{t} \lambda_{k} d\left(\tilde{p}_{k}, \tilde{p}\right)
$$

where $\tilde{p}_{k}=\left(\tilde{u}_{k}, \tilde{v}_{k}\right)=\left(\left[a_{k}, b_{k}\right],\left[c_{k}, d_{k}\right]\right)$.

Suppose $\tilde{P}$ has been ranked in descending order, that is $\lambda_{k} d\left(\tilde{p}_{k}, \tilde{p}\right) \geq \lambda_{k+1} d\left(\tilde{p}_{k+1}, \tilde{p}\right)$, where $\tilde{p}=(\tilde{u}, \tilde{v})=$ $([a, b],[c, d])$.

It is clear that $\tilde{p}_{1}$ is the element with the furthest weighted distance from $\tilde{P}$. Replace $\tilde{P}_{1}$ by $\tilde{P}$.

Let $\tilde{P}^{\prime}=\left\{\tilde{p}_{1}^{\prime}, \tilde{p}_{2}^{\prime}, \ldots, \tilde{p}_{t}^{\prime}\right\}$, where $\tilde{p}_{k}^{\prime}=\left(\tilde{u}_{k}^{\prime}, \tilde{v}_{k}^{\prime}\right)=$

$$
\begin{aligned}
\left(\left[a_{k}^{\prime}, b_{k}^{\prime}\right],\left[c_{k}^{\prime}, d_{k}^{\prime}\right]\right), \tilde{p}_{k}^{\prime} & =\left\{\begin{array}{c}
\tilde{p}, k=1 \\
\tilde{p}_{k}, k \neq 1
\end{array} .\right. \text { Then, } \\
S^{\prime} & =\sum_{k=1}^{t} \lambda_{k} d\left(\tilde{p}_{k}^{\prime}, \tilde{p}^{\prime}\right)
\end{aligned}
$$

where $\tilde{p}^{\prime}=\left(\tilde{u}^{\prime}, \tilde{v}^{\prime}\right)=\left(\left[a^{\prime}, b^{\prime}\right],\left[c^{\prime}, d^{\prime}\right]\right)$.

Now, the new weighted distance $S^{\prime}$ of $\tilde{P}^{\prime}=\left\{\tilde{p}_{1}^{\prime}, \tilde{p}_{2}^{\prime} \cdots \tilde{p}_{t}^{\prime}\right\}$ is less than $S$.

Theorem 1. Under (7) and (8), we have $S^{\prime}<S$.

Proof: According to (7) and (8) and Definition 8, we have

$$
\begin{aligned}
\tilde{p} & =\sum_{k=1}^{t} \lambda_{k} \tilde{p}_{k}=\left(\sum_{k=1}^{t} \lambda_{k} \tilde{u}_{k}, \sum_{k=1}^{t} \lambda_{k} \tilde{v}_{k}\right) \\
& =\left(\left[\sum_{k=1}^{t} \lambda_{k} \tilde{a}_{k}, \sum_{k=1}^{t} \lambda_{k} \tilde{b}_{k}\right],\left[\sum_{k=1}^{t} \lambda_{k} \tilde{c}_{k}, \sum_{k=1}^{t} \lambda_{k} \tilde{d}_{k}\right]\right) \\
& =([a, b],[c, d])
\end{aligned}
$$

and

$$
\begin{aligned}
\tilde{p}^{\prime} & =\sum_{k=1}^{t} \lambda_{k} \tilde{p}_{k}^{\prime}=\left(\sum_{k=1}^{t} \lambda_{k} \tilde{u}_{k}^{\prime}, \sum_{k=1}^{t} \lambda_{k} \tilde{v}_{k}^{\prime}\right) \\
& =\left(\left[\sum_{k=1}^{t} \lambda_{k} \tilde{a}_{k}^{\prime}, \sum_{k=1}^{t} \lambda_{k} \tilde{b}_{k}^{\prime}\right],\left[\sum_{k=1}^{t} \lambda_{k} \tilde{c}_{k}^{\prime}, \sum_{k=1}^{t} \lambda_{k} \tilde{d}_{k}^{\prime}\right]\right) \\
& =\left(\left[a^{\prime}, b^{\prime}\right],\left[c^{\prime}, d^{\prime}\right]\right)
\end{aligned}
$$

Then,

$$
\begin{aligned}
S^{\prime} & =\sum_{k=1}^{t} \lambda_{k} d\left(\tilde{p}_{k}^{\prime}, \tilde{p}^{\prime}\right) \\
& =\lambda_{1} d\left(\tilde{p}_{1}^{\prime}, \tilde{p}^{\prime}\right)-\lambda_{1} d\left(\tilde{p}_{1}, \tilde{p}^{\prime}\right)+\sum_{k=1}^{t} \lambda_{k} d\left(\tilde{p}_{k}, \tilde{p}^{\prime}\right) \\
& =\lambda_{1} d\left(\tilde{p}, \tilde{p}^{\prime}\right)-\lambda_{1} d\left(\tilde{p}_{1}, \tilde{p}^{\prime}\right)+\sum_{k=1}^{t} \lambda_{k} d\left(\tilde{p}_{k}, \tilde{p}^{\prime}\right)
\end{aligned}
$$


Consequently,

$$
\begin{aligned}
d\left(\tilde{p}, \tilde{p}^{\prime}\right)= & \left|a-a^{\prime}\right|^{2}+\left|b-b^{\prime}\right|^{2}+\left|c-c^{\prime}\right|^{2}+\left|d-d^{\prime}\right|^{2} \\
= & \left|\lambda_{1}\left(a-a_{1}\right)\right|^{2}+\left|\lambda_{1}\left(b-b_{1}\right)\right|^{2}+\left|\lambda_{1}\left(b-c_{1}\right)\right|^{2} \\
& +\left|\lambda_{1}\left(b-d_{1}\right)\right|^{2}=\lambda_{1}^{2} d\left(\tilde{p}_{1}, \tilde{p}\right)
\end{aligned}
$$

and

$$
\begin{gathered}
\lambda_{1} d\left(\tilde{p}_{1}, \tilde{p}^{\prime}\right)=\lambda_{1}\left[\left|a_{1}-a+a-a^{\prime}\right|^{2}+\left|b_{1}-b+b-b^{\prime}\right|^{2}\right. \\
\left.\quad+\left|c_{1}-c+c-c^{\prime}\right|^{2}+\left|d_{1}-d+d-d^{\prime}\right|^{2}\right] \\
=\lambda_{1}\left[(1+\lambda)^{2}\left|a_{1}-a\right|^{2}+(1+\lambda)^{2}\left|b_{1}-b\right|^{2}\right. \\
\left.\quad+(1+\lambda)^{2}\left|c_{1}-c\right|^{2}+(1+\lambda)^{2}\left|d_{1}-d\right|^{2}\right] \\
=\lambda_{1}(1+\lambda)^{2} d\left(\tilde{p}_{1}, \tilde{p}\right)
\end{gathered}
$$

It follows that

$$
\begin{aligned}
\sum_{k=1}^{t} \lambda_{k} d\left(\tilde{p}_{k}, \tilde{p}^{\prime}\right)= & \sum_{k=1}^{t} \lambda_{k}\left(\left|a_{k}-a^{\prime}\right|^{2}+\left|b_{k}-b^{\prime}\right|^{2}+\left|c_{k}-c^{\prime}\right|^{2}\right. \\
& \left.\quad+\left|d_{k}-d^{\prime}\right|^{2}\right) \\
= & \sum_{k=1}^{t} \lambda_{k}\left(\left|a_{k}-a+a-a^{\prime}\right|^{2}+\left|b_{k}-b+b-b^{\prime}\right|^{2}\right. \\
& \left.+\left|c_{k}-c+c-c^{\prime}\right|^{2}+\left|d_{k}-d+d-d^{\prime}\right|^{2}\right) \\
= & \sum_{k=1}^{t} \lambda_{k}\left[\left(\left|a_{k}-a\right|^{2}+\left|b_{k}-b\right|^{2}+\left|c_{k}-c\right|^{2}\right.\right. \\
& \left.+\left|d_{k}-d\right|^{2}\right)+\left(\left|a-a^{\prime}\right|^{2}+\left|b-b^{\prime}\right|^{2}\right. \\
& \left.\left.+\left|c-c^{\prime}\right|^{2}+\left|d-d^{\prime}\right|^{2}\right)\right] \\
= & S+\lambda_{1}^{2} d\left(\tilde{p}, \tilde{p}_{1}\right)
\end{aligned}
$$

So,

$$
\begin{aligned}
S^{\prime} & =\lambda_{1}^{3} d\left(\tilde{p}, \tilde{p}_{1}\right)-\lambda_{1}\left(1+\lambda_{1}\right)^{2} d\left(\tilde{p}, \tilde{p}_{1}\right)+S+\lambda_{1}^{2} d\left(\tilde{p}, \tilde{p}_{1}\right) \\
& =S-\lambda_{1}\left(1+\lambda_{1}\right) d\left(\tilde{p}, \tilde{p}_{1}\right)
\end{aligned}
$$

Thus, in this case, we have $S^{\prime}<S$.

Based on Theorem 1, if we replace the element with the mean value of the set, a smaller weighted distance will be obtained. Moreover, the weighted distance will be close to zero if we continue to use this strategy.

Theorem 2. Let $S^{(0)}=S$ be the initial weighted distance, and $\tilde{P}^{(0)}=\tilde{P}$ be the original set. Suppose $\left\{S^{(r)}\right\}$ and $\left\{\tilde{P}^{(k)}\right\}$ are the corresponding new weighted distances sequence and set sequence, respectively, we can get $\lim _{l \rightarrow \infty} S^{(l)}=0$ after using the above method $l$ times.
Proof: By Theorem 1, suppose we know that

$$
\begin{gathered}
\lambda_{1} d\left(\tilde{p}, \tilde{p}_{1}\right)=\max _{k}\left\{\lambda_{k} d\left(\tilde{p}, \tilde{p}_{k}\right)\right\} \\
\lambda_{1} d\left(\tilde{p}, \tilde{p}_{1}\right) \geq \frac{1}{t} \sum_{k=1}^{t} \lambda_{k} d\left(\tilde{p}, \tilde{p}_{k_{i}}\right)=\frac{S}{t}
\end{gathered}
$$

so

$$
\begin{aligned}
S^{(1)} & =S-\lambda_{1}\left(1+\lambda_{1}\right) d\left(\tilde{p}, \tilde{p}_{1}\right) \\
& \leq S-\lambda_{1} d\left(\tilde{p}, \tilde{p}_{1}\right) \\
& =S-\frac{S}{t} \\
& =\left(1-\frac{1}{t}\right) S
\end{aligned}
$$

Repeat the above method, we obtain

$$
S^{(l+1)} \leq S^{(l)}-\frac{S^{(l)}}{t}=\left(1-\frac{1}{t}\right) S^{(l)} \leq\left(1-\frac{1}{t}\right)^{l} S
$$

Therefore, $\lim _{l \rightarrow \infty} S^{(l)}=0$, and this completes the proof for Theorem 2.

According to Theorem 3, we obtain Corollary 1.

Corollary 1. Let $\tilde{S}^{(k)}=\left\{\tilde{s}_{1}^{(k)}, \tilde{s}_{2}^{(k)}, \ldots, \tilde{s}_{t}^{(k)}\right\}(k=1,2$, $\ldots, t)$ be the set generated by the above alter strategy for $k$ times and $\tilde{s}^{(k)}$ be the average value, then

$$
\lim _{k \rightarrow \infty} d\left(\tilde{s}_{i}^{(k)}, \tilde{s}^{(k)}\right)=0, \quad i=1,2, \ldots, t
$$

From the above analysis, we can apply the altered method to the consensus reaching process of the FMAGDM problem.

Theorem 3. Let $\tilde{P}^{(k)(0)}=\tilde{P}^{(k)}=\left(\tilde{p}_{i j}^{k}\right)_{n \times m}(k=1,2$, $\ldots, t)$ be IVIF matrices, where $\tilde{p}_{i j}^{k}=\left(u_{i j}^{k}, v_{i j}^{k}\right)$, and let $\tilde{P}^{(0)}=\tilde{P}=\left(\tilde{p}_{i j}\right)_{n \times m}$ be the group decision matrix using an IVIFWA operator, where $\tilde{p}_{i j}=\left(u_{i j}, v_{i j}\right)$. Assume $\tilde{P}^{(k)(h+1)}$ and $\tilde{P}^{(h+1)}$ are the corresponding matrices through implementing the following change method: for some fixed $p$ and $q$, assume $\lambda_{\mu} d\left(\tilde{p}_{p q}^{u(h)}, \tilde{p}_{i j}\right)=\max _{k} \lambda_{k}\left(\tilde{p}_{p q}^{k(h)}, \tilde{p}_{i j}\right)$, then

$$
\tilde{p}_{p q}^{k(h+1)}= \begin{cases}\tilde{p}_{p q}^{(h+1)} & \text { if } k=u \\ \tilde{p}_{p q}^{k(h+1)} & \text { otherwise }\end{cases}
$$

We have

$$
\begin{gathered}
\lim _{h \rightarrow \infty} d\left(\tilde{p}_{i j}^{k(h)}, \tilde{p}_{i j}^{(h)}\right)=0 \\
i=1,2, \ldots, n ; \quad j=1,2, \ldots, m ; \quad k=1,2, \ldots, t
\end{gathered}
$$

Proof: Similar to Theorems 1 and 2, we obtain Theorem 3.

Theorems 1-3 can be considered as a tool to decide which decision makers should change their own ideas. So, 


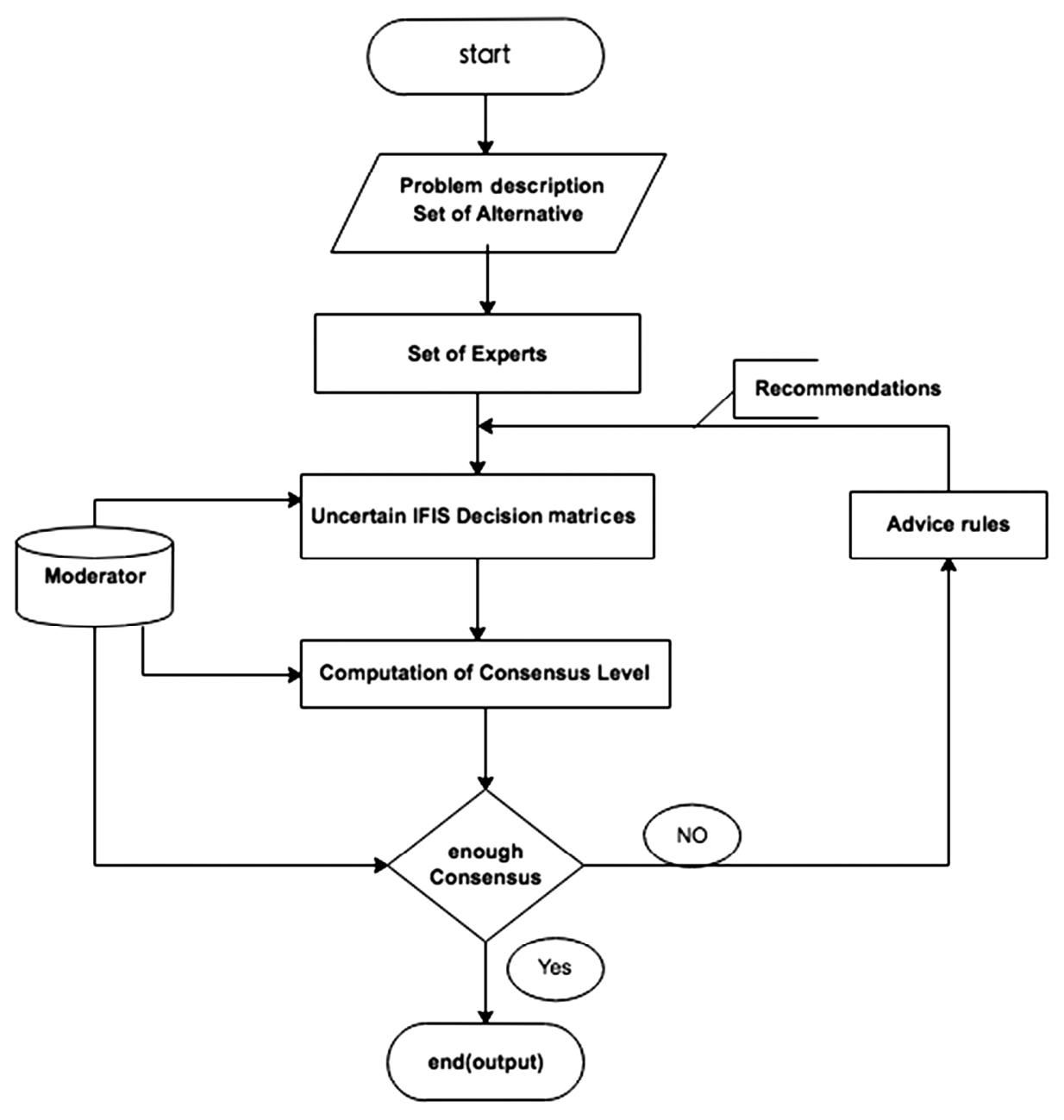

Figure 1. The MAGDM consensus reaching process.

a consensus reaching process for FMAGDM problems can be given. In each step of this procedure, the moderator estimates the current consensus degree among the decision makers applying a consensus measure. Once the consensus level obtained cannot satisfy the threshold level, we can use a process to reach consensus, which is shown in Fig. 1.

Algorithm 1. Consensus reaching process

Step 1. Let $l=0$ and $\tilde{P}^{(k)(0)}=\tilde{P}^{(k)}$, where $k=1,2, \ldots, t$.

Step 2. Calculate the group decision matrix using the formula: $\tilde{P}^{(l)}=\left(\tilde{p}_{i j}^{(l)}\right)_{n \times m}$, and compute $\tilde{p}_{i j}^{(l)}=$ $U L W A\left(\tilde{p}_{i j}^{1(l)}, \tilde{p}_{i j}^{2(l)}, \cdots, \tilde{p}_{i j}^{t(l)}\right)$ according to Definition 3.

Step 3. Compute the conflict degree of $\tilde{P}^{(k)(l)}$ using $\theta\left(\tilde{P}^{(k)(l)}\right)=(1 / n m) \sum_{i=1}^{n} \sum_{j=1}^{m} d\left(\tilde{p}_{i j}^{k(l)}, \tilde{p}_{i j}^{(l)}\right)$. If all $G C I\left(\tilde{P}^{(k)(l)}\right) \leq \delta$, go to step 7; otherwise, go to step 4 .

Step 4. Calculate the weighted distance matrix $S^{(l)}=$ $\left(s_{i j}^{(l)}\right)_{n \times m}$, where $s_{i j}^{(l)}=\sum_{k=1}^{t} \lambda_{k} d\left(\tilde{p}_{i j}^{k(l)}, \tilde{p}_{i j}^{(l)}\right)$.

Step 5. Seek out the position of the maximum elements of $S^{(l)}$, and let $s_{i_{l} j_{l}}^{(l)}=\max _{i, j}\left\{s_{i j}^{(l)}\right\}$.
Step 6. Decide which expert should modify his preferences. Find $u$ subject to

$$
\lambda_{u} d\left(\tilde{p}_{i_{l} j_{l}}^{u(l)}, \tilde{p}_{i_{l} j_{l}}^{(l)}\right)=\max _{k} \lambda_{k} d\left(\tilde{p}_{i_{l} j_{l}}^{k(l)}, \tilde{p}_{i_{l} j_{l}}^{(l)}\right)
$$

let

$$
\tilde{p}_{i j}^{k(l+1)}= \begin{cases}\tilde{p}_{i_{l} j_{l}}^{(l)} & \text { if } k=u \\ \tilde{p}_{i_{l} j_{l}}^{k(l)} & \text { otherwise }\end{cases}
$$

Set $l=l+1$, then return to step 2 .

Step \%. Output $\tilde{P}^{k(l)}$ and compute the final group decision matrix $\tilde{P}^{(l)}=\left(\tilde{p}_{i j}^{(l)}\right)$.

Algorithm 1 can increase the consensus level of the FMAGDM problem. The convergence of Algorithm 1 is presented in Theorem 4.

Remark. The threshold values $\delta$ for group consensus index should be given at first. To increase the convergence rate, two or more elements can be changed in one round.

Theorem 4. Let $\tilde{P}^{k}=\left(\tilde{p}_{i j}^{k}\right)(k \in T)$ be $t$ IVIF decision matrices. Let $\left\{\tilde{P}^{k(l)}\right\}$ and $\left\{\tilde{P}^{(l)}\right\}$ be the matrices sequences coming from Algorithm 1, respectively. Then, 
Table 1

The Failure Components of UAV Power System

\begin{tabular}{|l|l|l|l|}
\hline Serial Number & Fault Component & Failure Mode & Failure Effect \\
\hline$A_{1}$ & $\begin{array}{l}\text { Fuel electronic } \\
\text { controller failure }\end{array}$ & Control accuracy reduction & $\begin{array}{l}\text { Engine cannot be } \\
\text { accurately controlled }\end{array}$ \\
\hline$A_{2}$ & Engine fault & Engine thrust reduction & $\begin{array}{l}\text { Failing to complete the task } \\
\text { due to lack of engine drive }\end{array}$ \\
\hline$A_{3}$ & $\begin{array}{l}\text { Inlet and outlet } \\
\text { device failure }\end{array}$ & $\begin{array}{l}\text { When the engine works, the } \\
\text { speed rises and falls }\end{array}$ & Engine stall fault \\
\hline$A_{4}$ & $\begin{array}{l}\text { Installation joint } \\
\text { bolt failure }\end{array}$ & bolt crack & $\begin{array}{l}\text { Mounting bolts failed and the } \\
\text { aircraft lost thrust }\end{array}$ \\
\hline$A_{5}$ & Loosening of engine mounting & The engine is shaking & Engine vibration overrun fault \\
\hline
\end{tabular}

we can get $\lim _{l \rightarrow \infty} d\left(\tilde{P}^{k(l)}, \tilde{P}^{(l)}\right)=0$. In particular, there exists an integer $L$, for any $l>L, d\left(\tilde{P}^{k(l)}, \tilde{P}^{(l)}\right) \leq \delta$, where $\delta$ is a initial conflict level.

Proof: It is clear that Theorem 4 can be proved by Theorem 3.

Based on Theorem 4, Algorithm 1 can be implemented by using finite times, thus becoming effective.

Once the consensus level among experts has been obtained, we can get a new group decision matrix $\tilde{P}=\left(\tilde{p}_{i j}\right)$, where $\tilde{p}_{i j}=\left(\tilde{u}_{i j}, \tilde{v}_{i j}\right)$. In this section, a FMAGDM procedure for evaluating mutually exclusive alternatives is proposed. Then, the fuzzy suitability attribute values for the alternatives are calculated, and the rank of the alternatives is obtained based on the given attributes and IVIF weighted average (IVIFWA) operator.

\section{Evaluating the Power System of UAV}

As presented in Table 1, the common power system faults of a certain type of UAV mainly includes $A_{1}$ : fuel electronic controller failure, $A_{2}$ : engine fault, $A_{3}$ : inlet and outlet device failure, $A_{4}$ : installation joint bolt failure, $A_{5}$ : loosening of engine mounting and so on.

Suppose there is a reliable evaluate panel composed of three experts, the reliability assessment should be based on FMAGDM analysis of the following intuitionistic fuzzy matrices after comprehensively considering the decision attributes, in which $c_{1}$ is the frequency of failure mode, $c_{2}$ is the risk degree, and $c_{3}$ is the degree of detection, the weight vector of the decision attributes is $\omega=(0.3576,0.3257,0.3172)$. The IVIF decision matrices of the evaluation towards the failure components of UAV power system given by the experts are listed as follows:

$$
\begin{aligned}
& \tilde{P}^{1}=\tilde{P}^{1(1)}=\left(\begin{array}{ccc}
([0.25,0.34],[0.42,0.45]) & ([0.23,0.35],[0.44,0.65]) & ([0.41,0.5],[0.24,0.35]) \\
([0.46,0.58],[0.24,0.35]) & ([0.18,0.26],[0.66,0.71]) & ([0.13,0.35],[0.34,0.45]) \\
([0.53,0.75],[0.13,0.18]) & ([0.33,0.45],[0.46,0.51]) & ([0.33,0.45],[0.24,0.38]) \\
([0.22,0.35],[0.41,0.52]) & ([0.23,0.45],[0.34,0.45]) & ([0.61,0.71],[0.22,0.25]) \\
([0.71,0.75],[0.14,0.25]) & ([0.63,0.71],[0.18,0.24]) & ([0.33,0.45],[0.44,0.51])
\end{array}\right) \\
& \tilde{P}^{2}=\tilde{P}^{2(1)}=\left(\begin{array}{ccc}
([0.36,0.52],[0.35,0.38]) & ([0.53,0.65],[0.24,0.35]) & ([0.46,0.52],[0.24,0.36]) \\
([0.36,0.51],[0.24,0.35]) & ([0.18,0.26],[0.66,0.71]) & ([0.13,0.25],[0.34,0.48]) \\
([0.53,0.78],[0.13,0.24]) & ([0.63,0.72],[0.16,0.21]) & ([0.53,0.61],[0.24,0.34]) \\
([0.26,0.35],[0.41,0.52]) & ([0.43,0.45],[0.34,0.52]) & ([0.51,0.61],[0.28,0.35]) \\
([0.56,0.65],[0.28,0.35]) & ([0.71,0.76],[0.18,0.20]) & ([0.33,0.39],[0.48,0.52])
\end{array}\right) \\
& \tilde{P}^{3}=\tilde{p}^{3(1)}=\left(\begin{array}{ccc}
([0.32,0.54],[0.41,0.45]) & ([0.35,0.47],[0.44,0.52]) & ([0.43,0.5],[0.32,0.44]) \\
([0.32,0.48],[0.48,0.52]) & ([0.28,0.32],[0.47,0.52]) & ([0.26,0.35],[0.34,0.45]) \\
([0.43,0.55],[0.14,0.18]) & ([0.26,0.42],[0.46,0.51]) & ([0.36,0.45],[0.34,0.38]) \\
([0.22,0.35],[0.36,0.52]) & ([0.28,0.45],[0.34,0.45]) & ([0.64,0.71],[0.22,0.25]) \\
([0.63,0.75],[0.17,0.25]) & ([0.63,0.71],[0.22,0.24]) & ([0.26,0.37],[0.54,0.61])
\end{array}\right)
\end{aligned}
$$


The detailed application for the given algorithms is carried out in the following steps:

\section{Stage 1: Consensus process reaching}

As the choice of the most possible power system fault depends on the preference of a group of experts, the interactive consensus analysis is indispensable. First, without loss of generality, suppose the weight vector of the decision experts is $\lambda=(0.32,0.24,0.44)$.

Step 1. Calculate original group decision matrix based on $\tilde{p}_{1}, \tilde{p}_{2}$ and $\tilde{p}_{3}$ using Definition 3 with a specified matrix $\tilde{p}^{(1)}$, where

$$
\tilde{P}^{(1)}=\left(\begin{array}{cccc}
([0.3072,0.4712],[0.3988,0.4332]) & ([0.3548,0.4748],[0.3920,0.5208]) & ([0.4308,0.5048],[0.2752,0.3920]) \\
([0.3744,0.5192],[0.3456,0.4248]) & ([0.2240,0.2864],[0.5764,0.6264]) & ([0.1872,0.3260],[0.3400,0.4752]) \\
([0.4860,0.6692],[0.1344,0.1944]) & ([0.3712,0.5016],[0.3880,0.4380]) & ([0.3912,0.4884],[0.2840,0.3704]) \\
([0.2296,0.3500],[0.3880,0.5200]) & ([0.3000,0.4500],[0.3400,0.4668]) & ([0.5992,0.6860],[0.2344,0.2740]) \\
([0.6388,0.7260],[0.1868,0.2740]) & ([0.6492,0.7220],[0.1976,0.2304]) & ([0.2992,0.4004],[0.4936,0.5564])
\end{array}\right)
$$

Step 2. Calculate the initial conflict degree induces for each expert using the consensus measure, and then we get $\theta\left(\tilde{P}^{1(1)}\right)=0.0119, \quad \theta\left(\tilde{P}^{2(1)}\right)=0.0347, \quad \theta\left(\tilde{P}^{3(1)}\right)=0.0096$

If the initial value is fixed at $\delta=0.01$, then the newly gained consensus indices should be compared to this threshold. If $\tilde{p}_{1}, \tilde{p}_{2}$ and $\tilde{p}_{3}$ fail to reach the initial conflict degree, a consensus reaching process should be continued. Otherwise, it can stop here.

Obviously, it can be seen that $\tilde{P}^{1}$ and $\tilde{P}^{2}$ do not fulfil the initial consensus level, so a consensus reaching process should be used and the sequences must be modified through continuous implementing Algorithm 1 until the threshold consensus level is acquired.

Step 3. Compute the weighted distance matrix

$$
S^{(1)}=\left(\begin{array}{lll}
0.0116 & 0.0444 & 0.0038 \\
0.0269 & 0.0211 & 0.0062 \\
0.0144 & 0.0701 & 0.0137 \\
0.0009 & 0.0067 & 0.0070 \\
0.0097 & 0.0023 & 0.0066
\end{array}\right)
$$

Step 4. It can be seen that the position of the maximum element is in the $(3,2)$.

As

$$
\begin{aligned}
d\left(\tilde{p}_{32}^{2(1)}, \tilde{p}_{32}\right) & =0.2187>d\left(\tilde{p}_{32}^{3(1)}, \tilde{p}_{32}\right) \\
& =0.0295>d\left(\tilde{p}_{32}^{1(1)}, \tilde{p}_{32}\right)=0.0148
\end{aligned}
$$

so $\tilde{p}_{32}^{2(1)}$ should be changed by $\tilde{p}_{32}^{(1)}$, that is,

$$
\tilde{p}_{32}^{2(2)}=\tilde{p}_{32}^{(1)}=([0.3712,0.5016],[0.3880,0.4380])
$$

Continue to apply Algorithm 1, the detailed modified processes are as follows:

(i) $\tilde{p}_{32}^{2(2)}=\tilde{p}_{32}^{(1)}=([0.3712,0.5016],[0.3880,0.4380])$;

(ii) $\tilde{p}_{12}^{2(3)}=\tilde{p}_{12}^{(2)}=([0.3548,0.4748],[0.3920,0.5208])$;

(iii) $\tilde{p}_{21}^{3(4)}=\tilde{p}_{21}^{(3)}=([0.3744,0.5192],[0.3456,0.4248])$; (iv) $\tilde{p}_{31}^{3(5)}=\tilde{p}_{31}^{(4)}=([0.4860,0.6692],[0.1344,0.1944])$;

(v) $\tilde{p}_{33}^{2(6)}=\tilde{p}_{33}^{(5)}=([0.3912,0.4884],[0.2840,0.3704])$.

After modification, the conflict degrees for each modified decision matrix $\tilde{P}^{k(6)}(k=1,2,3)$ become

$\theta\left(\tilde{P}^{1(6)}\right)=0.0071, \quad \theta\left(\tilde{P}^{2(6)}\right)=0.0100, \quad \theta\left(\tilde{P}^{3(6)}\right)=0.0052$

which gives the initial consensus level. The detailed computation steps are ignored here.

Overall, the improved individual decision matrices and the final group decision matrix are obtained after 6 iterations. Once the consensus level among experts has been obtained, we get a new group decision matrix $\tilde{P}=\left(\tilde{p}_{i j}\right)$, where $\tilde{p}_{i j}=\left(\tilde{u}_{i j}, \tilde{v}_{i j}\right)$. Then, the rank of the alternatives is obtained based on the given attributes.

Compute the value $\tilde{z}_{i}$ of $A_{i}(i=1,2, \ldots, 5)$ using IVIFWA operator as

$$
\begin{aligned}
& \tilde{z}_{1}=([0.3482,0.3510],[0.3693,0.4684]) \\
& \tilde{z}_{2}=([0.2747,0.3884],[0.3977,0.4914]) \\
& \tilde{z}_{3}=([0.3966,0.5498],[0.2846,0.3516]) \\
& \tilde{z}_{4}=([0.3697,0.4891],[0.3237,0.4247]) \\
& \tilde{z}_{5}=([0.5345,0.6214],[0.2876,0.3494])
\end{aligned}
$$

Then, based on Definition 2, calculate the score $S_{i}$ of $\tilde{z}_{i}(i=1,2, \ldots, 5)$ as follows:

$$
\begin{aligned}
& S_{1}=-0.0692, \quad S_{2}=-0.1130, \quad S_{3}=0.1551, \\
& S_{4}=0.0552, \quad S_{5}=0.2595
\end{aligned}
$$

Then, based on (1) and (2), we can get the overall ranking of $A_{i}(i=1,2, \ldots, 5)$ as

$$
A_{5} \succ A_{3} \succ A_{4} \succ A_{1} \succ A_{2}
$$


The severity level of the above, mentioned failure components can be ordered as:

Loosening of engine mounting $>$ Inlet and outlet device failure $>$ Installation joint bolt failure $>$ Fuel electronic controller failure $>$ Engine fault

Through the field investigation and analysis, the evaluation results are in line with the actual situation suggesting the reliability level of UAV power system can be improved.

\section{Conclusion}

In this paper, an improved FMAGDM is proposed to evaluate the fault of the power system of the UAVs, which can show the prediction ability of our predictors. In this method, the attribute values are given by IVIF numbers, which can represent the possible occurrence rate and the impossible occurrence rate in the circumstance where the labelled data is absent. Moreover, this approach is characterized by introducing a new algorithm to achieve a desirable consensus in group decision-making. The main features of the given method are as follows. It is not necessary to change much information of the decision matrices given by the experts in the consensus reaching process, which can improve the accuracy of decision with the lower computational load.

In summary, the proposed method has potential to help reducing the failure rate of drones and improving the reliability and stability of drones through giving designer and maintainers more useful information from quantitative group decisions. The theoretical analysis is not limited to the fault detection problem, but rather more practical problems. In future, according to the decision model presented, a web-based software tool capable of working simultaneously with different levels of precision and granularity spaces will be built. The developed software will provide experts a useful tool for ranking alternatives.

\section{Acknowledgement}

The authors wish to express their gratitude to anonymous referees for valuable comments and suggestion. This study is jointly funded by the National Natural Science Fund of China (No. 61673285); Found Project of Science and Technology Department in Sichuan Province-Research and implementation of the medium and small civilian UAV airworthiness monitoring platform and key technology (2018JZ0066); Research project on technical Road map of key industries in Chengdu - Technical Road map of unmanned aerial vehicle industry in Chengdu (2016YJ01-00009-JH); And The innovation team project of the Sichuan Provincial Education Department-Application and research of rotor unmanned aerial vehicle in industrial field (17TD0001).

\section{References}

[1] M. Dong, K. Ota, M. Lin, Z. Tang, S. Du, and H. Zhu, UAVassisted data gathering in wireless sensor networks, Journal of Supercomputing, 70(3), 2014, 1142-1155.
[2] Yuehao Yan, Chengda LI, Rong Wang, Research on key technologies of unmanned aerial vehicle intelligent four shaft rotor, ICCWAMTIP, 2014, 495-449.

[3] Z.M. Fadlullah, D. Takaishi, H. Nishiyama, N. Kato, and R. Miura, A dynamic trajectory control algorithm for improving the communication throughput and delay in UAV-aided networks, IEEE Network, 1(30), 2016, 100-105.

[4] I. Jawhar, N. Mohamed, J. Al-Jaroodi, and S. Zhang, A framework for using unmanned aerial vehicles for data collection in linear wireless sensor networks, Journal of Intelligent 8 Robotic Systems, 74(1-2), 2013, 437.

[5] K. Li, W. Ni, X. Wang, R.P. Liu, S.S. Kanhere, and S. Jha, Energy-efficient cooperative relaying for unmanned aerial vehicles, IEEE Transactions on Mobile Computing, 15(6), 2016, 1377-1386.

[6] Y. Saleem, M.H. Rehmani, and S. Zeadally, Integration of cognitive radio technology with unmanned aerial vehicles: issues, opportunities, and future research challenges, Journal of Network and Computer Applications, 50, 2015, 15-31.

[7] S. Say, H. Inata, J. Liu, and S. Shimamoto, Priority-based data gathering framework in UAV-assisted wireless sensor networks, IEEE Sensors Journal, 16(14), 2016, 5785-5794.

[8] G. Steinbauer, A survey about faults of robots used in RoboCup, RoboCup 2012: Robot Soccer World Cup XVI, Springer, Berlin, Heidelberg, 2013, 344-355.

[9] R. Isermann, Model-based fault-detection and diagnosis-status and applications, Annual Reviews in Control, 29, 2005, 71-85.

[10] Z. Lv, L. Zheng, X. Liang, and X. Liang, A fuzzy multiple attribute decision making method based on possibility degree, Journal of Intelligent $\&$ Fuzzy Systems, 31, 2016, 787-794.

[11] S.M. Chen and Z.C. Huang, Multiattribute decision making based on interval-valued intuitionistic fuzzy sets, PSO techniques, and evidential reasoning methodology, IEEE Transactions on Fuzzy Systems, 23(6), 2017, 1905-1916.

[12] C.Y. Tsao and T.Y. Chen, A projection-based compromising method for multiple criteria decision analysis with intervalvalued intuitionistic fuzzy information, Applied Soft Computing, 45, 2016, 207-223.

[13] J.Y. Chai, J.N.K. Liu, and Z.S. Xu, A rule-based group decision model for warehouse evaluation under interval-valued intuitionistic fuzzy environments, Expert Systems with Applications, 40(6), 2016, 1959-1970.

[14] S.P. Wan, J. Xu, and J.Y. Dong, Aggregating decision information into interval-valued intuitionistic fuzzy numbers for heterogeneous multi-attribute group decision making, Knowledge-Based Systems, 113, 2016, 155-170.

[15] L.E. Wang, H.C. Liu, and M.Y. Quan, Evaluating the risk of failure modes with a hybrid MCDM model under intervalvalued intuitionistic fuzzy environments, Computers $\&$ Industrial Engineering, 102, 2016, 175-185.

[16] N. Howard, Paradoxes of rationality: Theory of metagames and political behavior (Cambridge, MA: MIT Press, 1971).

[17] N. Howard, Confrontation analysis: how to win operations other than war, Office of the assistant secretary of defense Washington DC command and control research program (CCRP), 1999

[18] D.M. Kilgour, K.W. Hipel, and L.P. Fang, The graph model for conflicts, Automatica, 23, 1987, 41-55.

[19] J.P. Xu and Z.B. Wu, A discrete consensus support model for multiple attribute group decision making, Knowledge-Based Systems, 24(8), 2011, 1196-1202.

[20] Y.J. Zhang, A.J. Li, and T. Fung, Using GIS and multiple criteria decision analysis for conflict resolution in land use planning, Procedia Environmental Sciences, 13, 2010, 2264-2273.

[21] Y.C. Dong, G.Q. Zhang, W.C. Hong, and Y.F. Xu, Consensus models for AHP group decision making under row geometric mean prioritization method, Decision Support Systems, 49(3), 2010, 281-289.

[22] F. Chiclana, F. Mata, L. Martínez, E. Herrera-Viedma, and S. Alonso, Integration of a consistency control module within a consensus model, International Journal of Uncertainty, Fuzziness and Knowledge-Based Systems, 16(1), 2008, 35-53.

[23] F. Herrera, S. Alonso, F. Chiclana, and E. Herrera-Viedma, Computing with words in decision making: foundations, trends 
and prospects, Fuzzy Optimization and Decision Making, 8, 2009, 337-364.

[24] D. Ben-Aieh and Z.F. Chen, Linguistic group decision-making: opinion aggregation and measures of consensus, Fuzzy Optimization and Decision Making, 5(4), 2006, 371-386.

[25] K.G. Zografos, G.M. Vasilakis, and I.M. Giannouli, Methodological framework for developing decision support systems (DSS) for hazardous materials emergency response operations, Journal of Hazardous Materials, 71(1), 2000, 503-521.

[26] R.O. Parreiras, P.Y. Ekel, J.S.C. Martini, and R.M. Palhares, A flexible consensus scheme for multiple criteria group decision making under linguistic assessments, Information Sciences, 180(7), 2010, 1075-1089.

\section{Biographies}

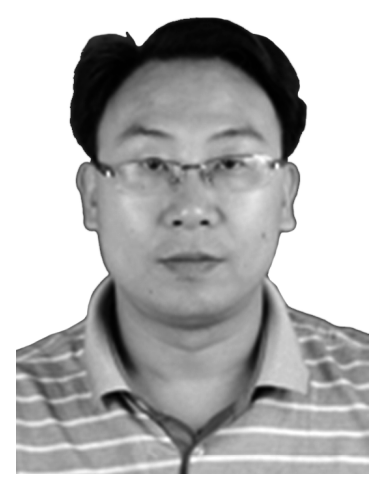

Yuehao Yan is national outstanding scientific and technical worker, associate professor, executive president of UAV Industrial Technology Research Institute in Chengdu Technological University, executive director of the key Laboratory of flight control application and technology of UAV system in Sichuan Regular Higher Education Institutions. He is a Ph.D. student in school of aeronautics Northwestern Polytechnical University. His research interests lay in the fields of flight control technology, performance evaluation of UAVs, monitor and navigation system for UAVs. He has been involved in a number of projects, like National Natural Science Fund Project, Fund Project of Education department in Sichuan Provincial and Found Project of Science and Technology Department in Sichuan Province. As the first author, he has a lot of papers published in academic journals and conferences, some of which were indexed by SCI and EI.

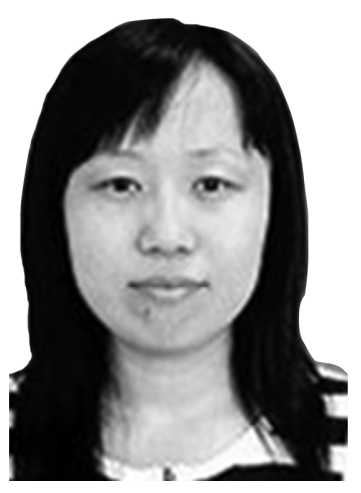

Zhiying Lv is Post doctor of University of Electronic Science and Technology of China, the teacher of Chengdu University of Information Technology. Her research interests lay in the fields of uncertain decision, machine learning and artificial intelligence methods. She has been involved in a number of projects, like National Natural Science Fund Project, Fund Project of Sichuan Provincial department of Education and Found Project of Science and Technology Department in Sichuan Province. She has published papers in academic journals and conferences, some of which were indexed by SCI and EI.

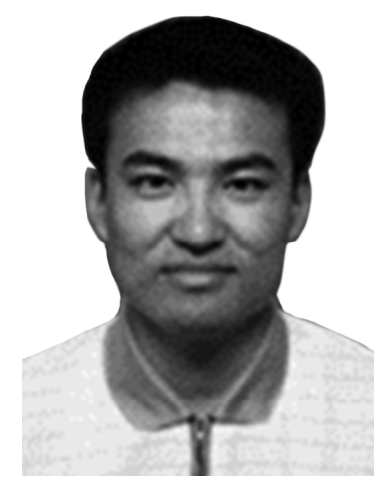

Ruiyu Zhang received his M.S degree in electronics and information engineering from Harbin Institute of Technology. He is currently an engineer with Beijing Institute of information technology. His research interests include deep learning, reinforcement learning, pattern recognition and application of artificial intelligence on UAV systems. He has been involved in a number of projects, like earth observation and navigation technology in the national high-tech research and development plan. He has published papers in geomatics and information science of Wuhan University and so on. 